\title{
Aplicación de vibraciones sobre la superficie dell dializador: Efecto en la dosis de heparina
}

\author{
Alberto Sánchez Martín - Belén Muñoz García - Mª Luz Sánchez Tocino - Silvia Villoria González - \\ Beatriz Hernández Olalla
}

Fundación Renal Iñigo Álvarez de Toledo. FRIAT. Centro "El Castañar" Béjar. Centro "Las Encinas" Ciudad Rodrigo. Salamanca

\section{Resumen}

El cebado del dializador realizado de manera adecuada, hace que se expandan los capilares correctamente y se impregne bien la membrana con la solución. Con el objetivo de facilitar el cebado del dializador se colocó junto al dializador un dispositivo que, de forma constante, realiza un movimiento de vibración en su interior lo que favorece la correcta eliminación de microburbujas y el reparto homogéneo de la heparina.

Se incluyeron en este estudio a 8 pacientes que tenían requerimientos de heparina mayores de los habituales y que sus dializadores al finalizar la sesión presentaban capilares coagulados. Durante trece sesiones se aplicó el dispositivo reduciendo la dosis de heparina a la tercera sesión si el dializador se mantenía limpio al finalizar ésta. Se realizó un registro fotográfico del estado del dializador al finalizar la sesión.

La dosis media de heparina inicial fue de 3500 $\pm 534,522$ u.i. y al finalizar el estudio fue de $2562,50 \pm 728,869$ u.i., los índices de hemólisis, $\mathrm{Kt} / \mathrm{V}$, hemoglobina y resto de parámetros analíticos

Correspondencia:

Alberto Sánchez Martín

Fundación Renal Iñigo Álvarez de Toledo

C/ Arco del Monte núm. 3 Bajo

37700 Béjar (Salamanca)

castanar@friat.es no evidencian diferencias. El registro fotográfico mostró dializadores limpios al finalizar el estudio. Con este método hemos conseguido que el circuito se mantenga limpio con dosis de heparina inferiores y sin alteración de los parámetros analíticos.

PALABRAS CLAVE:

- CEBADO DEL DIALIZADOR

- DOSIS DE HEPARINA

- HEMÓLISIS

- COAGULACIÓN

- HEMODIÁLISIS

\section{Application of vibrations to the dialyser surface. Effect on the heparin dose}

\section{Abstract}

When the dialyser is suitably primed, the capillaries expand correctly and the membrane is well impregnated with the solution. In order to facilitate the priming of the dialyser, a device was placed next to the dialyser which constantly produces a vibration inside it to favour the correct elimination of microbubbles and uniform distribution of the heparin.

This study included 8 patients with higher than normal heparin requirements and whose dialysers at the end of the session showed coagulated capillaries. Over thirteen sessions, the device was applied reducing the heparin dose on the third session if the dialyser was still clean at the end of that session. A photographic 
record was kept of the state of the dialyser at the end of the session.

The mean initial dose of heparin was $3500 \pm 534.522$ i.u. and at the end of the study it was $2562.50 \pm 728.869$ i.u., the haemolysis, Kt/V and haemoglobin indices and other analytical parameters did not show any differences. The photographic record showed clean dialysers once the study was complete. With this method we have managed to keep the circuit clean with lower doses of heparin and with no alteration of the analytical parameters.

\section{KEY WORDS:}

- DIALYSER PRIMING

- HEPARIN DOSE

- HAEMOLYSIS

- COAGULATION

- HAEMODIALYSIS

\section{Introducción}

Es necesario un ajuste preciso de la dosis de heparina durante la sesión de hemodiálisis para que dializador y cámaras queden libres de coágulos ${ }^{1}$. Se ha comprobado la influencia que tiene sobre la dosis de heparina administrada, el correcto cebado del circuito ${ }^{2-6}$ y un adecuado retorno de sangre por parte del personal de enfermería ${ }^{4}$. Incidencias como la detención de la bomba arterial durante un tiempo excesivo, problemas de flujo del acceso vascular, ultrafiltración elevada o la recirculación del circuito implican un riesgo de coagulación del sistema ${ }^{6,7}$. Los lavados con suero fisiológico, muy utilizados en sesiones sin heparina, permiten evaluar el estado del dializador y las cámaras $5^{5,8,9,10}$.
En ocasiones, se dan pequeños golpes sobre el dializador o las cámaras, que físicamente, lo que hacen es provocar una vibración en su interior. Estos golpes durante el cebado, los lavados o el retorno, desprenden los restos adheridos a la membrana, y eliminan eventuales micro-burbujas. Si se forman coágulos en el circuito de diálisis es necesario cambiar la zona afectada, el dializador, o incluso todo el circuito ${ }^{1,6}$ y en algunos casos, no se podrá retornar la sangre, con la consiguiente pérdida para el paciente.

Hace algunos años se estudió el efecto de las vibraciones externas aplicadas en la pared abdominal en la eficiencia de la diálisis peritoneal, concluyendo que aumentaba el aclaramiento de manera significatival1,12. Nuestro trabajo intenta evaluar el efecto de la vibración que provocamos al golpear el dializador, mantenida de forma constante, durante toda la sesión de hemodiálisis. Nos planteamos por tanto, los siguientes objetivos:

- Evaluar la influencia que tienen las vibraciones aplicadas al dializador durante la hemodiálisis.

- Valorar su efecto en la dosis de heparina administrada al paciente.

- Objetivar los posibles efectos adversos

\section{Material y método}

Se construyen varios dispositivos provistos de (figura 1):

- Micro-motor eléctrico con eje excéntrico. Utilizados habitualmente en el interior de los gamepads y miniaturizados en teléfonos móviles.

- Interruptor de bajo voltaje para marquetería

- Adaptador de corriente AC/CC de 5v

- Cinta elástica, compresor o "tortor".

- Pinza kocher

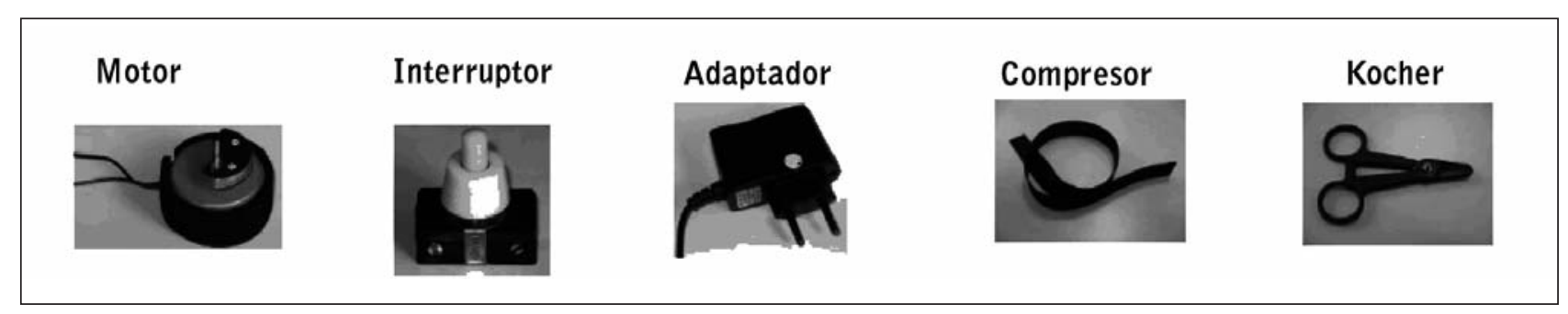

Figura 1: Elementos utilizados en la realización del dispositivo 
- Material adicional: estaño, soldador de estaño, cable de dos hilos, cinta termoretráctil, multímetro, gafas y guantes de seguridad, cámara fotográfica.

Seleccionamos como muestra a 8 pacientes con necesidades de mayores dosis de heparina cuyo circuito, en ocasiones, presenta capilares coagulados. Sus características son: siete son portadores de FAVI, con tiempos de hemostasia superiores a 10 minutos y uno es portador de catéter yugular disfuncionante. Seis pacientes utilizan heparina sódica no fraccionada frente a dos que tienen pautada enoxoparina. Dos pacientes utilizan dializadores PES190DH y un paciente PES210DH de Nipro ${ }^{\circledR}$, un paciente utiliza el dializador FX80M y cuatro pacientes el HF80S del Fresenius ${ }^{\circledR}$.

Realizamos un estudio prospectivo experimental durante un periodo de un mes que engloba trece sesiones de hemodiálisis convencional. Se solicitó el consentimiento informado verbal a los pacientes.

Durante la fase de montaje y cebado del monitor se coloca y activa el micro-motor, que estará en contacto permanente con el dializador hasta que finaliza el tratamiento. Queda fijado mediante el compresor y la pinza, como muestra la figura 2. Se realiza el cebado siguiendo el protocolo de nuestra unidad con $1000 \mathrm{cc}$ de suero fisiológico con 5000 u.i. de heparina (Prontopri$\mathrm{me}^{\circledR}$ ). La zona venosa permanece hacia arriba durante el tratamiento como aconseja la literaura, ${ }^{90}$. Al finalizar cada sesión se fotografían dializador y cámaras. Durante este periodo no se modifica ningún otro parámetro de la pauta de hemodiálisis. Se mantienen constantes el flujo de bomba, tiempo de la sesión, tratamiento con eritropoyetina, hierro etc.

Si el circuito queda limpio durante tres sesiones consecutivas se reajusta la dosis de heparina y se realiza una sesión posterior, sin colocar el dispositivo. Se realiza una analítica previa y posterior al periodo de estudio, incluyendo es esta última el índice de hemólisis de la muestra y de un grupo control, ya que habitualmente no disponemos de este parámetro.

El análisis estadístico de los datos recogidos de las gráficas de enfermería y las analíticas, se realizó con el programa SPSS 15.0 para Windows. Los resultados de las variables se expresaron como media \pm desviación típica. Las variables cuantitativas se analizaron con $\mathrm{t}$ de Student para muestras relacionadas. Se considero significativo un valor de $p<0,05$.

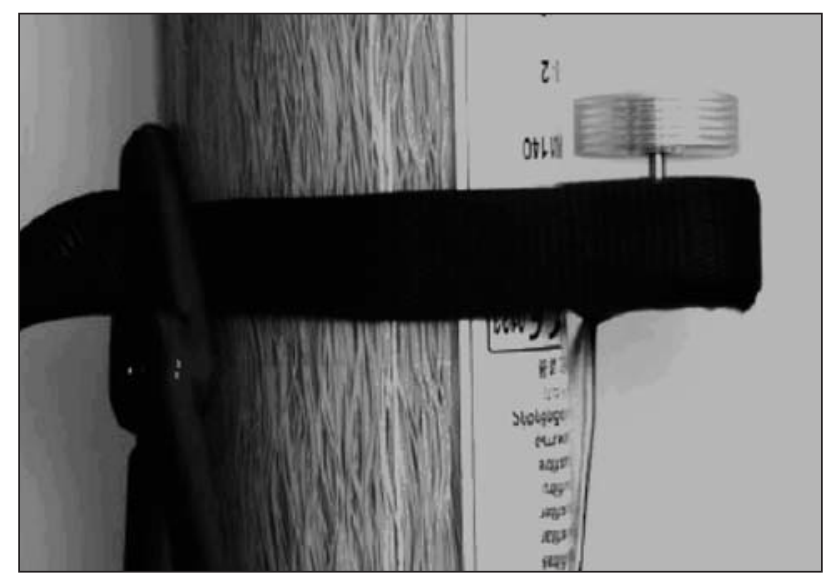

Figura 2: Detalle del montaje y fijación del dispositivo al dializador

\section{Resultados}

La edad media de los pacientes fue de 76 años (rango 57-87). La dosis media de heparina con la que iniciamos el estudio fue $3500 \pm 534,522$ u.i. Al finalizar el estudio la dosis media resultante fue $2562,50 \pm 728,869$ u.i. con una $p$ de 0,002 (tabla 1, figura 3, figura 4). En los índices de hemólisis de la muestra, Kt/V, hemoglobina y resto de parámetros analíticos no evidencian diferencias estadísticamente significativas. Tampoco se evidencian diferencias significativas entre los índices de hemolisis de la muestra y del grupo control.

\begin{tabular}{|c|c|c|c|}
\hline n=8 & $\begin{array}{c}\text { Inicio del } \\
\text { estudio }\end{array}$ & Fin del estudio & P-valor \\
\hline Heparina & $3500 \pm 534,522$ & $2562,50 \pm 728,869$ & 0,002 \\
\hline Kt/V & $1,6800 \pm 0,18928$ & $1,7162 \pm 0,18776$ & n.s. \\
\hline Hemoglobina & $12,1125 \pm 1.04668$ & $121625 \pm 0,57306$ & n.s. \\
\hline $\begin{array}{c}\text { Índice } \\
\text { hemólisis }\end{array}$ & $4 \pm 2,16025$ & $4 \pm 2,58199$ & n.s. \\
\hline
\end{tabular}

Tabla 1: Resultados estadísticos de los datos más relevantes del estudio

La secuencia fotográfica de uno de los casos estudiados se muestra en la figura 5: de izquierda a derecha se muestra el estado del dializador en la hemodiálisis previa al estudio (hd 0), en la última hemodiálisis del 
periodo analizado ( $h d 13$ ) y en una sesión realizada a posteriori, en la que se mantiene la dosis ajustada de heparina pero sin colocar el dispositivo (hd14).

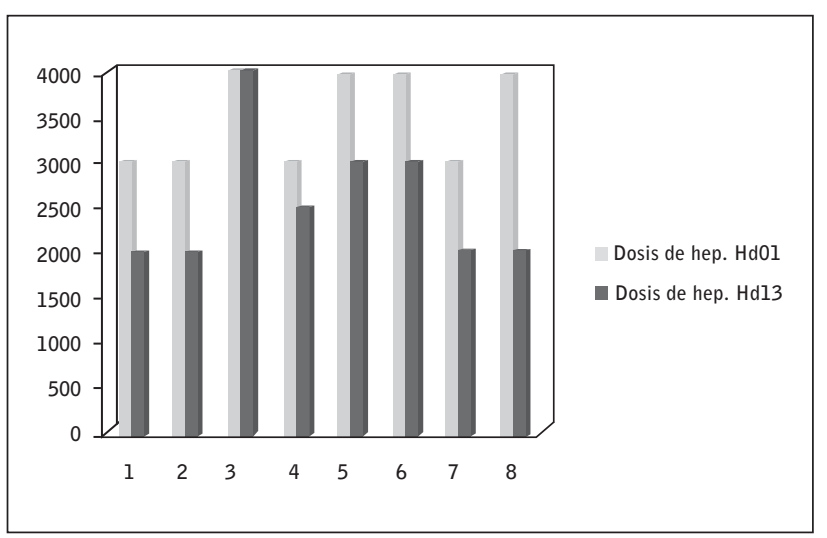

Figura 3: Dosis de heparina administrada a cada uno de los pacientes en la diálisis inicial y final del estudio

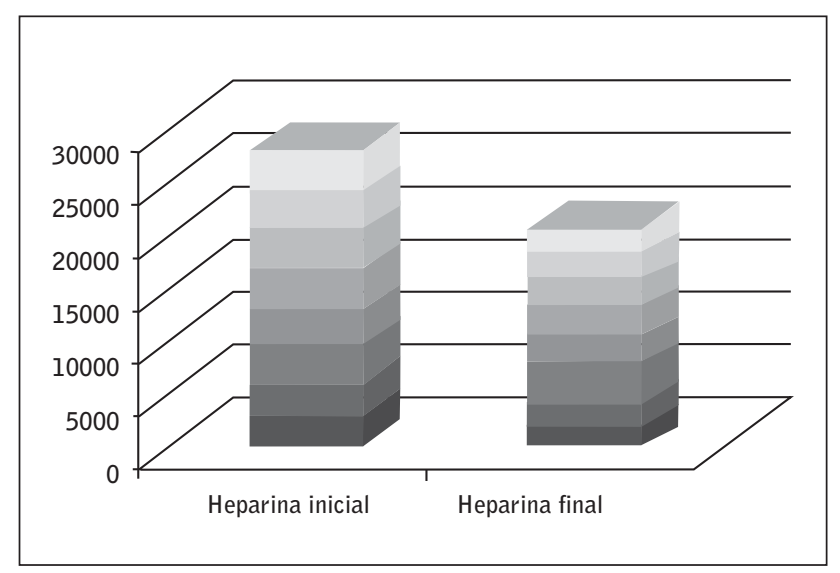

Figura 4:Dosis total de heparina administrada al principio del estudio $\mathrm{y}$ al término del periodo.

\section{Discusión}

El método de fijación del dispositivo, aunque artesanal, resultó eficaz, seguro y no invasivo. La carga de trabajo adicional para enfermería no fue elevada ya que solamente se utilizó un dispositivo por enfermero/a y turno que, una vez co locado, no requería ninguna otra intervención.

La reducción de dosis de heparina administrada al paciente es significativa. Esto se debe a los efectos que parece producir la vibración:
1. En la fase de purgado del circuito, permite un reparto homogéneo de la heparina de cebado, a lo largo de toda la superficie del dializador y una correcta expansión de los capilares. Además facilita la eliminación de las eventuales microburbujas de aire que pudieran quedar atrapadas en los capilares y no apreciarse a simple vista.

2. Durante el tratamiento, evita que se creen depósitos en la parte superior del dializador, que podrían obstruir los capilares, lo que disminuiría la superficie útil del dializador y por tanto la eficacia dialítica.

En el caso del paciente portador de un catéter temporal disfuncionante con problemas habituales de flujo, no se consiguió reducir la dosis de heparina (caso 3 del figura 3); pero durante el mes del estudio sólo precisó un recambio de línea venosa, mientras que en el mes previo se registraron un cambio completo del sistema y dos ocasiones en las que se redujo el tiempo de diálisis por riesgo de coagulación (grandes coágulos en cámaras).

Los índices de hemólisis son bajos (entre 1 y 12), tanto en las muestras, como en el grupo control, sin diferencias significativas. Tampoco se registran diferencias en el resto de controles analíticos realizados.

Respecto a la secuencia fotográfica, en la primera columna se puede observar la situación inicial de tres de los casos ( $h d 0$ ), en las que se aprecia que algunos de los capilares quedan sucios. Las fotografías de la columna del centro reflejan el estado del dializador en la última sesión realizada con el dispositivo (hd13). En ellas se puede observar que el filtro está limpio y que no existen coágulos en la cámara venosa. Para confirmar que la reducción de dosis de heparina no se debía de un incorrecto ajuste inicial, realizamos la sesión posterior al estudio ( $h d 14$ ), con la dosis de heparina ajustada, pero sin el dispositivo. La última columna refleja esta situación. El dializador presentó capilares coagulados y suciedad en cámaras en todos los casos, necesitando volver a las dosis previas de heparina en las siguientes sesiones.

No se han observado episodios de sangrado post-hemodiálisis de las FAVIs durante el periodo, presentándose dos episodios en el mes anterior en uno de los casos. Algunos de los pacientes parecen coagular mejor, pero no se han objetivado diferencias en los tiempos de hemostasia. 


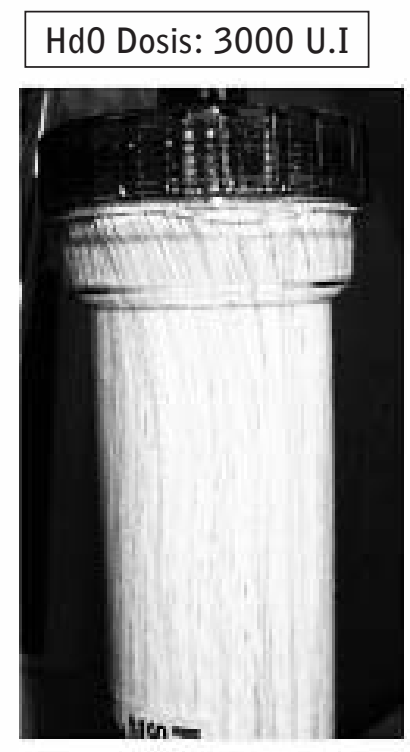

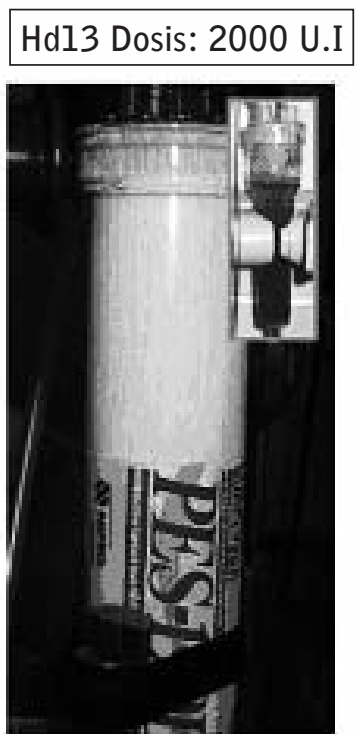

Hd14 Dosis: 2000 U.I

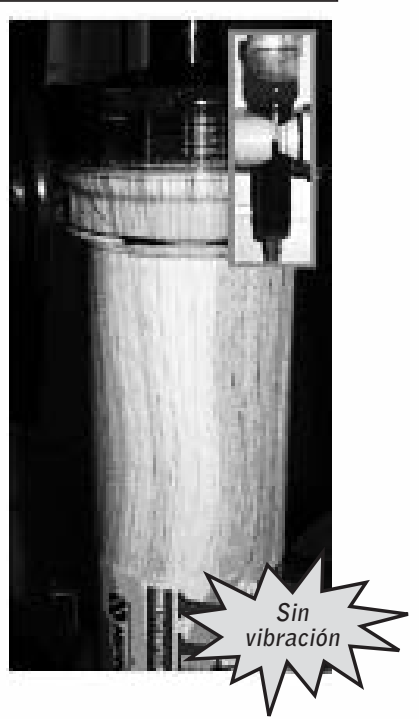

Figura 5: Secuencia fotográfica de un caso

Nos hemos encontrado con las siguientes dificultades:

- No poseemos métodos para evaluar la modificación sobre el flujo laminar teórico que debe existir dentro del dializador.

- No disponemos de un medidor de vibraciones para conocer la intensidad en $\mathrm{Hz}$ que aplica nuestro motor al dializador, ya que si esa vibración resultase muy elevada quizás sí provocaría hemólisis. En diálisis peritoneal se han utilizado frecuencias de 2 a $6 \mathrm{~Hz}$ y 5 a $10 \mathrm{~mm}$ de amplitud con buenos resultados ${ }^{11,12}$.

Al tratarse de un estudio piloto, con una muestra pequeña, y de características concretas, no pueden extrapolarse las conclusiones al resto de pacientes, pero se puede deducir que una ligera vibración, aplicada de forma constante al dializador, disminuye la formación de coágulos en el sistema, permitiendo reducir la dosis de heparina administrada al paciente, sin que se evidencie un aumento de hemólisis, a corto plazo. A la espera de disponer de datos a largo plazo, se podría plantear la posibilidad de implementar un dispositivo similar al que hemos utilizado integrado en el brazo de la máquina de diálisis.

\section{Agradecimientos}

Al resto de personal, en especial al Dr. Miguel Terleira por su implicación en el estudio y a Vicente Ginés, técnico de mantenimiento de nuestros centros, que participó activamente en la fase de planificación y resolvió las incidencias. A la Dra. $\mathrm{M}^{\mathrm{a}}$ Victoria Hernández, del laboratorio del Hospital Universitario de Salamanca, por analizar personalmente las muestras de hemólisis.

\section{Bibliografía}

1. Manual de protocolos y procedimientos de actuación de enfermería nefrológica SEDEN. Cuidados de enfermería durante la sesión. 2001. Disponible en www.revistaseden.org/files/art93_1.pdf Fecha de consulta Abril 2009.

2. Del Valle MJ y cols. Necesidades de Heparina en Hemodiálisis. Influencia del Purgado, la dosis inicial y el Flujo Sanguíneo. Comunicaciones presentadas al XVIII Congreso Nacional de la Sociedad Española de Enfermería Nefrológica. La Coruña, octubre 1993. 
3. Ojeda Ramírez $M^{a} D$, García Hita S, García Pérez $A M^{a}$. Intervención de enfermería en la reducción de la dosis de heparina con la membrana AN69-ST. Libro XXXII Congreso Nacional de la Sociedad Española de Enfermería Nefrológica. Cádiz 3-6 0ctubre 2007. Pág. 267-272.

4. Gago C, Gruss E y cols. Estudio comparativo de diferentes Pautas de Recuperación al final de la Diálisis: Influencia en la Dosis de Heparina. Madrid. Póster SEDEN 1997.

5. Carral A, Sola T, Higuera C, Begines A, Donat A, Lavin A. Diálisis sin heparina. Comunicaciones presentadas al X Congreso Nacional de la Sociedad Española de Enfermería Nefrológica. Las Palmas de Gran Canaria, 1985.

6. Andreu L. 500 cuestiones que plantea el cuidado del paciente renal. Barcelona. Masson, 1997.p125127.

7. La calidad en la diálisis, ¿Una cuestión de enfermería? Manuscrito de la Sesión Educacional Gambro en el XVIII Congreso Sociedad Española de Enfermería Nefrológica. La Coruña.1993.

8. Daugirdas JT. Manual de diálisis. Masson 1996. p.192-207.

9. Nissenson. Manual de diálisis 4 ed. Elselvier Masson. 2008. p.175 p.225-227.

10. Pascual R, Martín C, Delgado 0. Hemodiálisis sin anticoagulantes. Disponible en http://www.revistaseden.org/files/Hemodiálisis sin anticoagulantes.pdf Fecha de consulta: Abril 2009.

11. Rudoy J, Kohan R, Ben-Ari J. Externally Applied Abdominal Vibration as a Method for Improving Efficiency in Peritoneal Dialysis. Nephron 1987; 46:364-366.

12. Tan XY, Liu FY, Duan SB. Effects of nitroprusside and vibration on peritoneal transport of solutes in continuous ambulatory peritoneal dialysis patients. Bulletin of Hunan Medical University. 2000; 25(4):357-60. 